\title{
Uso del software "calmay" como herramienta de apoyo en el aprendizaje del sistema numérico maya
}

\author{
André Rivera \\ Universidad Central del Ecuador \\ asrivera@uce.edu.ec
}

Recibido: 9 - febrero - 2028 / Aceptado: 31 - julio - 2018

\section{Resumen}

En el presente documento se presenta la experiencia de aula llevada a cabo en abril de 2018 en la Unidad Educativa "Cardenal Carlos María de la Torre", con un grupo de 42 estudiantes del 10mo año EGB. En la institución se propuso enseñar a los estudiantes el Sistema Numérico Maya además de incluir al software "CalMay" (una calculadora maya) como una herramienta de apoyo en el proceso de enseñanza aprendizaje, ya que este puede ser instalado en ordenadores con sistema operativo Windows y Smartphones Android 4.4 o superior.

Palabras clave: sistema numérico maya, TIC, etnomatemática, etnociencias.

\section{Abstract}

This document presents the classroom experience carried out in April 2018 at the "Cardenal Carlos María de la Torre" Educational Unit, with a group of 42 students from the 10th Year of Primary Education. At the institution it was proposed to teach students the Maya Numeric System in addition to including the software "CalMay" (a Mayan calculator) as a tool to support 
the learning process since it can be installed on computers with Windows operating system and Android Smartphones 4.4 or higher.

Keywords. maya numerical system, TIC, ethnomathematics, ethnosciences. 


\section{Contextualización}

$\mathrm{L}$ os mayas forman parte de las civilizaciones más antiguas del mundo, su origen data de hace unos 10 mil años, y tuvieron grandes descubrimientos y conocimientos en varias áreas. De todas ellas destacaremos a la matemática, cuyos estudios, experiencias y conocimientos les permitieron desarrollar su propio sistema numérico.

El sistema Maya, en comparación con el resto de culturas de América, es considerado como el más razonable y sostenible desde el punto de vista matemático-astronómico. Dentro de este sistema existen dos aspectos de gran importancia para la matemática que hace que el mismo sobresalga entre sistemas de otras culturas, los cuales son: el cero y el valor posicional. Otras culturas que ya establecieron un sistema de numeración, tales como la romana y egipcia, aún con todos sus estudios y avances en la matemática, no lograron establecer estos conceptos, tan solo en la cultura hindú existe la noción de estos dos saberes, pero, los mismos los descubrieron hasta 300 años después que los mayas (Salazar de León, 2005).

Los mayas contaban con dos formas de numeración: los numerales geométricos o normales y los numerales en forma humana (antropomórficos), los cuales que eran representados por rostros antropomorfos y en algunos casos especiales se utilizaba todo el cuerpo (Salazar de León, 2005).

Esta variación de la numeración maya se le conoce actualmente como un sistema vigesimal por ser 20 su base, es decir, el valor que le corresponde a cada cantidad surge de la multiplicación de la cantidad por la potencia de base 20 .

La idea de establecer a este sistema como vigesimal nace del uso los dedos para contar, pues si utilizamos los dedos de las manos, ya contamos hasta el $10 \mathrm{y}$ si a eso le aumentamos los de los pies llegamos a 20, usamos a una persona para contar 20 (Fernández, 2013).

Por ejemplo, si quisiéramos representar el número 37 , necesitaríamos de una persona completa, las dos manos de otra persona, los dedos de su pie izquierdo y dos dedos de su pie derecho.

En el idioma maya, una persona y el número 20 llevan el mismo nombre, "JUN MAY" que se podría traducir como: una persona completa, una persona de 20.

Una gran hazaña de los mayas fue simplificar este sistema, siendo necesario tan solo 3 símbolos para la representación de un valor como lo son: puntos, rayas y el símbolo de la concha (Díaz \& Escobar, 2006).

\section{Referentes teórico-prácticos básicos}

- Sistema numérico maya

- Representación de los números mayas

- Operaciones matemáticas

- Adición de números mayas

- Sustracción de números mayas

- Producto de números mayas 


\section{Descripción general de la experiencia de aula}

En la Unidad Educativa "Cardenal Carlos María de la Torre" de la parroquia El Quinche, cantón Quito, se escogió a jóvenes de 10 mo año EGB, con un total de 42 estudiantes participantes.

Es inusual ver dentro de un aula de clase a un docente enseñar a sus alumnos el sistema numérico maya por lo cual, la primera reacción de los participantes fue de asombro e incertidumbre ya que era un tema desconocido totalmente por ellos.

Se llevó a los estudiantes al laboratorio de computación donde se realizó la clase, con ayuda de un proyector y un ordenador portátil se mostró a los jóvenes información clara y concisa sobre este tema, haciendo uso de texto e imágenes para evitar confusiones y generar un rápido aprendizaje en los participantes. Para comprender este sistema se presenta una dificultad media ya que usualmente los estudiantes están más familiarizados con el uso de números y no con símbolos como los que usaban los mayas.

Adicionalmente se presentó a los jóvenes el software "CalMay" como una herramienta de apoyo en el aprendizaje del sistema maya ya que el mismo permite realizar operaciones como suma, resta, multiplicación con números mayas. Por motivos de comodidad y mejor apreciación del software, este fue instalado en los ordenadores del laboratorio y en los smartphones de los participantes.

Una vez concluida la clase, se realizó una evaluación a los jóvenes en donde se les pidió resolver una cierta cantidad de ejercicios, 30 de ellos lo realizaron de manera tradicional, es decir, solo aplicando lo visto en clase, una hoja y un lápiz, los 12 restantes resolvieron la misma evaluación, pero tuvieron como recurso adicional el software cargado en el ordenador o su smartphone. Esta evaluación fue cronometrada con el fin de determinar cuál de los dos grupos podía resolverla en el menor tiempo posible.

La figura 1 muestra un grafica de los resultados obtenidos en la experiencia de aula donde, se puede revisar el tiempo que les tomó a cada estudiante resolver la evaluación, tanto usando el método tradicional, así como con la ayuda de la tecnología.

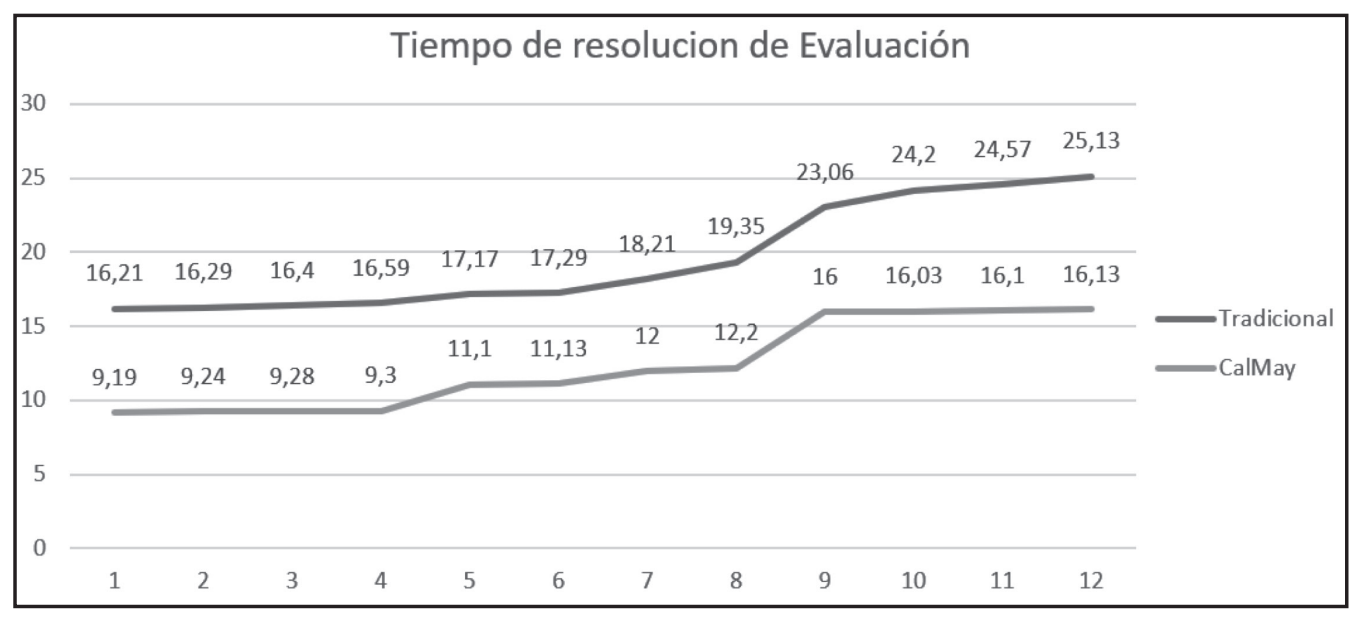

Figura 1. Resultados del tiempo de resolución de la evaluación. 


\section{Logros evidenciados}

Los estudiantes que utilizaron el software resolvieron la evaluación en casi la mitad del tiempo que los que no lo utilizaron.

Los participantes mostraron un gran interés por conocer más acerca de este tipo de saberes poco convencionales.

Se pudo generar un mejor entendimiento y aprendizaje en los estudiantes en un corto tiempo gracias a la implementación de tecnología para llevar a cabo la clase

\section{Dificultades evidenciadas}

Para llevar a cabo la clase se disponía de un tiempo limitado ya que el Sistema Numérico Maya no se encuentra dentro de un plan de clases o planificación de una asignatura en específico.

$\mathrm{Al}$ ser inusual encontrar este tema dentro en un aula de clase se pudo evidenciar al inicio que los participantes tenían ciertos inconvenientes para comprender otro sistema de numeración totalmente distinto al que están acostumbrados a utilizar.

\section{Reflexión final}

La educación en la actualidad presenta grandes desafíos a causa de la integración de ordenadores, smartphones, tabletas e internet como una herramienta de apoyo en el proceso de enseńanza aprendizaje, esto a su vez hace que cada uno de los estudiantes capte la información y produzca conocimiento de otra forma.

Métodos tradicionales u obsoletos como el dictado, realización de trabajos a mano e incluso la búsqueda de información en libros físicos en la biblioteca local se presentan como algo negativo e incluso desmotivante para los estudiantes, mucha información recolectada en todo el mundo se encuentra al alcance de un clic.

La solución a los problemas a los que enfrenta el docente con la educación actual no es forzar a sus alumnos a usar métodos de aprendizaje antiguos sino erradicarlos y buscar nuevas y mejores estrategias y métodos, haciendo uso de las TIC para enseñar además de desarrollar las herramientas digitales que sirvan de apoyo en cada clase.

Si vamos a proporcionar a un estudiante una herramienta digital, lo más adecuado sería que la misma sirva para aprender algo nuevo que no solo permita generar un aprendizaje sino también la investigación y generación de nuevos conocimientos.

En segundo lugar, se verificó que, al incorporar elementos históricos como el desarrollo de la matemática maya en Mesoamérica, el enfoque de la matemática toma un matiz humano, cronológico e identitario

\section{Referencias}

Covián, Olda. \& Cantoral, Ricardo. (2005). El papel del conocimiento matemático en la construcción de la vivienda tradicional: El caso de la cultura maya [archivo PDF]. Recuperado de: http://funes.uniandes.edu.co/5726/1/CantoralElpapelAlme2006.pdf 
Díaz, Nancy Dayana \& Escobar, Sandra Viviana. (2006). Articulación de actividades didácticas con algunos aspectos históricos de la cultura y matemática maya en el desarrollo del pensamiento espacial y sistemas geométricos del grado séptimo [archivo PDF]. Recuperado de: http://www.etnomatematica.org/publica/trabajos_grado/ articulacion_mayas.pdf

Fernández, Nicolás Luis. (2013). Sistema de numeración posicional del reino maya [archivo PDF]. Recuperado de: http://www.uco.es/users/malfegan/Comunes/ recursos-matematicos/Sistemas-numeracion/Sistema-de-numeracion-Maya.pdf

Morales, Leonel. (2007). Material de capacitación para ONGs sobre estándares educativos y matemática maya [archivo PDF]. Recuperado de: http://pdf.usaid.gov/ pdf_docs/Pnadq529.pdf

Salazar de León, Erwin Eduardo. (2005). Análisis comparativo de los conceptos matemáticos maya y kaxlan. El caso de las comunidades Santa Isabel y La Unión, Municipio de Chisec, Departamento de Alta Verapaz [archivo PDF]. Recuperado de: http://etnomatematica.org/trabgrado/ErwinSalazar.pdf

Yojcom, Domingo. (2013). La epistemología de la matemática maya: una construcción de conocimientos y saberes a través de prácticas [archivo PDF]. Recuperado de: http://www.etnomatematica.org/publica/trabajos_doctorado/tesis_maya.pdf

\section{Anexos}

Tabla 1. Estudiantes que usaron metodología tradicional

\begin{tabular}{|c|c|c|c|c|}
\hline $\mathrm{N}^{\circ}$ & $\begin{array}{l}\text { Nombre del Estu- } \\
\text { diante }\end{array}$ & Nivel & Metodología & Tiempo \\
\hline 1 & Alex Trejo & 10mo Año EGB & $\begin{array}{l}\text { Metodología tra- } \\
\text { dicional }\end{array}$ & $16^{\prime} 21 \mathrm{~min}$ \\
\hline 2 & Melanie Carrera & 10mo Año EGB & $\begin{array}{l}\text { Metodología tra- } \\
\text { dicional }\end{array}$ & $16^{\prime} 29 \mathrm{~min}$ \\
\hline 3 & Sasha Carvajal & 10mo Año EGB & $\begin{array}{l}\text { Metodología tra- } \\
\text { dicional }\end{array}$ & $16^{\prime} 40 \mathrm{~min}$ \\
\hline 4 & Antony Carrillo & 10mo Año EGB & $\begin{array}{l}\text { Metodología tra- } \\
\text { dicional }\end{array}$ & $16^{\prime} 59 \mathrm{~min}$ \\
\hline 5 & Isaac Solórzano & 10mo Año EGB & $\begin{array}{l}\text { Metodología tra- } \\
\text { dicional } \\
\end{array}$ & $17^{\prime} 17 \mathrm{~min}$ \\
\hline 6 & Brandon Sigcha & 10mo Año EGB & $\begin{array}{l}\text { Metodología tra- } \\
\text { dicional }\end{array}$ & $17^{\prime} 29 \mathrm{~min}$ \\
\hline 7 & $\begin{array}{l}\text { Sebastián Sán- } \\
\text { chez }\end{array}$ & 10mo Año EGB & $\begin{array}{l}\text { Metodología tra- } \\
\text { dicional }\end{array}$ & $18^{\prime} 21 \mathrm{~min}$ \\
\hline 8 & Kerly Cagua & 10mo Año EGB & $\begin{array}{l}\text { Metodología tra- } \\
\text { dicional }\end{array}$ & $19^{\prime} 35 \mathrm{~min}$ \\
\hline
\end{tabular}




\begin{tabular}{|c|c|c|c|c|}
\hline 9 & Melany Sánchez & 10mo Año EGB & $\begin{array}{l}\text { Metodología tra- } \\
\text { dicional }\end{array}$ & $23^{\prime} 06 \mathrm{~min}$ \\
\hline 10 & Juan Paredes & 10mo Ańo EGB & $\begin{array}{l}\text { Metodología tra- } \\
\text { dicional }\end{array}$ & $24^{\prime} 20 \mathrm{~min}$ \\
\hline 11 & Joseph Guerrero & 10mo Ańo EGB & $\begin{array}{l}\text { Metodología tra- } \\
\text { dicional }\end{array}$ & $24^{\prime} 57 \mathrm{~min}$ \\
\hline 12 & Jenifer Acosta & 10mo Ańo EGB & $\begin{array}{l}\text { Metodología tra- } \\
\text { dicional }\end{array}$ & $25^{\prime} 13 \mathrm{~min}$ \\
\hline 13 & Gerardo Quishpe & 10mo Año EGB & $\begin{array}{l}\text { Metodología tra- } \\
\text { dicional }\end{array}$ & $25^{\prime} 49 \mathrm{~min}$ \\
\hline 14 & Esteban Cevallos & 10mo Año EGB & $\begin{array}{l}\text { Metodología tra- } \\
\text { dicional }\end{array}$ & $26^{\prime} 01 \mathrm{~min}$ \\
\hline 15 & Kevin Puente & 10mo Ańo EGB & $\begin{array}{l}\text { Metodología tra- } \\
\text { dicional }\end{array}$ & $26^{\prime} 31 \mathrm{~min}$ \\
\hline 16 & Nicol Betancourt & 10mo Ańo EGB & $\begin{array}{l}\text { Metodología tra- } \\
\text { dicional }\end{array}$ & $27^{\prime} 01 \mathrm{~min}$ \\
\hline 17 & Samantha Peńa & 10mo Ańo EGB & $\begin{array}{l}\text { Metodología tra- } \\
\text { dicional }\end{array}$ & $27^{\prime} 13 \mathrm{~min}$ \\
\hline 18 & Melany Chávez & 10mo Año EGB & $\begin{array}{l}\text { Metodología tra- } \\
\text { dicional }\end{array}$ & $27^{\prime} 39 \mathrm{~min}$ \\
\hline 19 & Karen Arias & 10mo Año EGB & $\begin{array}{l}\text { Metodología tra- } \\
\text { dicional }\end{array}$ & $27^{\prime} 50 \mathrm{~min}$ \\
\hline 20 & Leidy Quishpe & 10mo Año EGB & $\begin{array}{l}\text { Metodología tra- } \\
\text { dicional }\end{array}$ & $28^{\prime} 04 \mathrm{~min}$ \\
\hline 21 & Erica Ríos & 10mo Ańo EGB & $\begin{array}{l}\text { Metodología tra- } \\
\text { dicional }\end{array}$ & $28^{\prime} 18 \mathrm{~min}$ \\
\hline 22 & $\begin{array}{l}\text { Cristina Echeve- } \\
\text { rria }\end{array}$ & 10mo Año EGB & $\begin{array}{l}\text { Metodología tra- } \\
\text { dicional }\end{array}$ & $28^{\prime} 43 \mathrm{~min}$ \\
\hline 23 & Nahomi Gualoto & 10mo Año EGB & $\begin{array}{l}\text { Metodología tra- } \\
\text { dicional }\end{array}$ & $29^{\prime} 02 \mathrm{~min}$ \\
\hline 24 & Lipsy Quishpe & 10mo Año EGB & $\begin{array}{l}\text { Metodología tra- } \\
\text { dicional }\end{array}$ & $30^{\prime} 31 \mathrm{~min}$ \\
\hline 25 & Wendy Cabrera & 10mo Ańo EGB & $\begin{array}{l}\text { Metodología tra- } \\
\text { dicional }\end{array}$ & $31^{\prime} 03 \mathrm{~min}$ \\
\hline 26 & María Pallo & 10mo Ańo EGB & $\begin{array}{l}\text { Metodología tra- } \\
\text { dicional }\end{array}$ & $32^{\prime} 28 \mathrm{~min}$ \\
\hline 27 & Joselin Cuascota & 10mo Año EGB & $\begin{array}{l}\text { Metodología tra- } \\
\text { dicional }\end{array}$ & $34^{\prime} 36 \mathrm{~min}$ \\
\hline
\end{tabular}




\begin{tabular}{|l|l|l|l|l|}
\hline 28 & Allison Sánchez & 10 mo Año EGB & $\begin{array}{l}\text { Metodología tra- } \\
\text { dicional }\end{array}$ & $36^{\prime} 05$ min \\
\hline 29 & $\begin{array}{l}\text { Evelyn Guayta- } \\
\text { rilla }\end{array}$ & 10 mo Año EGB & $\begin{array}{l}\text { Metodología tra- } \\
\text { dicional }\end{array}$ & $36^{\prime} 21$ min \\
\hline 30 & Wendy Farinango & 10 mo Año EGB & $\begin{array}{l}\text { Metodología tra- } \\
\text { dicional }\end{array}$ & $37^{\prime} 04$ min \\
\hline
\end{tabular}

Tabla 2. Estudiantes que usaron PC y smartphones

\begin{tabular}{|c|c|c|c|c|}
\hline $\mathrm{N}^{\circ}$ & $\begin{array}{l}\text { Nombre del Estu- } \\
\text { diante }\end{array}$ & Nivel & Metodología & Tiempo \\
\hline 1 & $\begin{array}{l}\text { Melany Contre- } \\
\text { ras }\end{array}$ & 10mo Año EGB & $\begin{array}{l}\text { Uso de PC / } \\
\text { Smartphone }\end{array}$ & $09^{\prime} 19 \mathrm{~min}$ \\
\hline 2 & Estefany Morillo & 10mo Año EGB & $\begin{array}{l}\text { Uso de PC / } \\
\text { Smartphone }\end{array}$ & $09^{\prime} 24 \mathrm{~min}$ \\
\hline 3 & Daniela Arteaga & 10mo Ańo EGB & $\begin{array}{l}\text { Uso de PC / } \\
\text { Smartphone }\end{array}$ & $09^{\prime} 28 \mathrm{~min}$ \\
\hline 4 & Cristel Palaguaray & 10mo Año EGB & $\begin{array}{l}\text { Uso de PC / } \\
\text { Smartphone }\end{array}$ & $09^{\prime} 30 \mathrm{~min}$ \\
\hline 5 & Juan Tuquerres & 10mo Año EGB & $\begin{array}{l}\text { Uso de PC / } \\
\text { Smartphone }\end{array}$ & $11^{\prime} 10 \mathrm{~min}$ \\
\hline 6 & Steven León & 10mo Año EGB & $\begin{array}{l}\text { Uso de PC / } \\
\text { Smartphone }\end{array}$ & $11^{\prime} 13 \mathrm{~min}$ \\
\hline 7 & Angeles Escorza & 10mo Año EGB & $\begin{array}{l}\text { Uso de PC / } \\
\text { Smartphone }\end{array}$ & $12^{\prime} 00 \mathrm{~min}$ \\
\hline 8 & Denisse Esparza & 10mo Año EGB & $\begin{array}{l}\text { Uso de PC / } \\
\text { Smartphone }\end{array}$ & $12^{\prime} 20 \mathrm{~min}$ \\
\hline 9 & Pamela Pazmiño & 10mo Año EGB & $\begin{array}{l}\text { Uso de PC / } \\
\text { Smartphone }\end{array}$ & $16^{\prime} 00 \mathrm{~min}$ \\
\hline 10 & Diana Rivas & 10mo Año EGB & $\begin{array}{l}\text { Uso de PC / } \\
\text { Smartphone }\end{array}$ & $16^{\prime} 03 \mathrm{~min}$ \\
\hline 11 & Jaime Flores & 10mo Año EGB & $\begin{array}{l}\text { Uso de PC / } \\
\text { Smartphone }\end{array}$ & $16^{\prime} 10 \mathrm{~min}$ \\
\hline 12 & Daniel Narváez & 10mo Año EGB & $\begin{array}{l}\text { Uso de PC / } \\
\text { Smartphone }\end{array}$ & $16^{\prime} 13 \mathrm{~min}$ \\
\hline
\end{tabular}

